\title{
Asociación de tuberculosis y alcohol en pacientes de una unidad de infecciosos
}

\author{
Andreu Ballester, J.C.*; Jaén Cervera, C. **; Segarra Castelló, L.*; Flores Cid, J.*; \\ Jaén Cervera, R.***; Cerdá Fayos, E.J.** \\ * Hospital Arnau de Vilanova de Valencia. \\ * Centro de Salud de Ruzafa Valencia. \\ *** Hospital General de Valencia. \\ Enviar correspondencia a: \\ Juan Carlos Andreu Ballester. C/ Juan Ramón Jimenez, 29-14a (46006-Valencia).Tfno: 963229473. e-mail: jcandreu@ono.com.
}

\section{RESUMEN}

Estudio prospectivo sobre la asociación de alcohol con la tuberculosis (TBC). En una población de 70 pacientes ingresados en la unidad de infecciosos con el diagnóstico de TBC pulmonar. Se valora el sexo, estado civil y el consumo de alcohol (gramos/día). Se comparan los resultados con un grupo control de 70 personas no tuberculosas, apareadas por edad, sexo y estado civil. Encontramos los siguientes resultados: El consumo de Alcohol se asocia a Tuberculosis pero sólo en los hombres (¿sesgo de respuesta?). Entre los bebedores la intensidad de consumo es mayor en los enfermos de TBC. Entre los enfermos de TBC no hay diferencias de intensidad de consumo por sexo, mientras que en los no tuberculosos, los hombres consumen más cantidad de alcohol. Hay una asociación clara entre TBC y alcohol en el grupo de los no casados (solteros, separados, viudos).

Palabras clave: Tuberculosis, alcohol, estado civil, sexo.

\section{SUMMARY}

Prospective study of the association between alcohol consumption and TBC. In a population of 70 patients studied in the Infectious Diseases Unit with the diagnosis of pulmonary TBC; the following parameters were evaluated: sex, civil status and the consumption of alcohol (grams/day). The results were compared with a control group of 70 non -TBC patients, matched by age, sex and civil status. Results: The consumption of alcohol is associated with Tuberculosis, only in the males sex (answer slant). Among the drinkers, the consumption of alcohol was larger in TBC patients. In TBC patients, there were no differences of consumption intensity in regard to sex, while in those without tuberculosis, the men consumed more quantity of alcohol. There is a clear association between TBC and alcohol in the group of those not married ( single, separated, widowers).

Key words: tuberculosis, alcohol, civil status, gender.

\section{INTRODUCCIÓN}

$\mathbf{L}$ a tuberculosis es una enfermedad infecciosa crónica producida fundamentalmente por el Mycobacterium tuberculosis. Es un bacilo ácidoalcohol resistente, aerobio estricto, de multiplicación lenta, que puede per-manecer en estado latente durante años, ya que la inmunidad celular que genera no impide su multiplicación y diseminación.

En 1990 se declararon en España 7.600 casos (tasa: 18,37/100.000); sin embargo, la cifra de enfermos estimada por consumo de tuberculostáticos es de unos
60/100.000(1). Ello hace de la tuberculosis un problema de salud pública. Se estima que en España se producen, cada año, entre 12000 y 14000 nuevos casos de enfermedad tuberculosa, de los que la mitad son altamente contagiosos (tasa: 30-35 casos/100.000 habitantes/año) (2).

Una de las complicaciones médicas apreciadas en el abuso del alcohol es la alteración en la regulación inmune que conlleva inmunodeficiencia, posiblemente por alteración en el equilibrio de las citokinas. Como consecuencia aumenta la susceptibilidad a la neumonía bacteriana y a la tuberculosis (3). 
Tomsen JL, estudió las autopsias forenses de 441 alcohólicos comparandolas con 255 no alcohólicos, objetivando que la Tuberculosis era más frecuente en alcohólicos, así como las neumonías lobares (4).

De todo lo anteriormente expuesto, existe una evidente asociación entre el consumo de alcohol, el nivel socioeconómico, la marginalidad, la malnutrición. Circunstancias especialmente concurrentes sobre todo en el sexo masculino. Por lo tanto el interés de este trabajo no solo se centra en la asociación alcohol tuberculosis, si no en el desarrollo de estrategias psicológicas y de apoyo a estos pacientes para facilitar el cumplimiento de la medicación y evitar así las recaídas.

\section{MATERIAL Y METODO}

El presente trabajo tiene como objetivo el estudio prospectivo de los pacientes tuberculosos y su relación con el alcohol.

La población la forman 70 enfermos ingresados con TBC en la unidad de infecciosos del Hospital Arnau de Vilanova de Valencia durante 1997-99.

Se determinan el sexo, edad, estado civil y, si son consumidores de alcohol, la cantidad ingerida (gr/día). Esta se valora con la fórmula: Gramos de alcohol=volumen de bebida $(\mathrm{ml}) \times$ grados de alcohol x 0,8/100.

La recogida de datos se realiza mediante: datos de laboratorio, encuesta directa a los pacientes (si beben o no alcohol y cantidad), historia clínica del enfermo.

Se ha relacionado el consumo de alcohol con el sexo y estado civil, en concreto entre dos grupos, el de casados y no casados (solteros, separados y viudos).

Así mismo se ha relacionado un grupo control, de 70 personas no tuberculosas, apareadas por edad, sexo y estado civil.

Se han utilizado: el T-test, o el de Kuskal-Wallis cuando las varianzas no eran homogéneas, para la comparación de medias y la chi-cuadrado para las variables cualitativas.

\section{RESULTADOS}

1. Entre los enfermos con TBC, beben alcohol 47 y no beben 23 frente a los 35 y 35 de los controles (Fig.1), con una OR (Odds ratio) $=2,04(\operatorname{ICO} 1,03-4,05) \mathrm{y}$ $p=0,0395$.
Fig. 1. Incidencia de alcohol en ambos grupos.

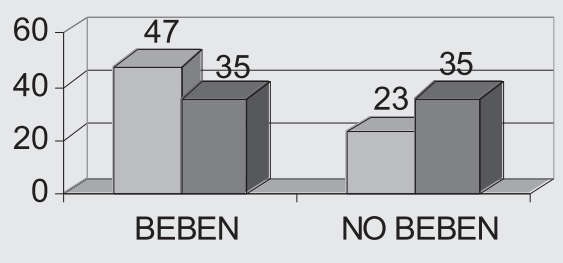

TBC $\square$ NO TBC

2. Al estudiar la asociación por sexos, encontramos que en los hombres la diferencia se mantiene estadísticamente significativa, con una $\mathrm{OR}=2,29$ (ICO 1,06-4,95) y $p=0,0344$ (Fig.2); mientras que no es así en las mujeres con una $O R=1,43(\operatorname{ICO} 0,20-10,55)$ y $p=0,6732$ (Fig.3).
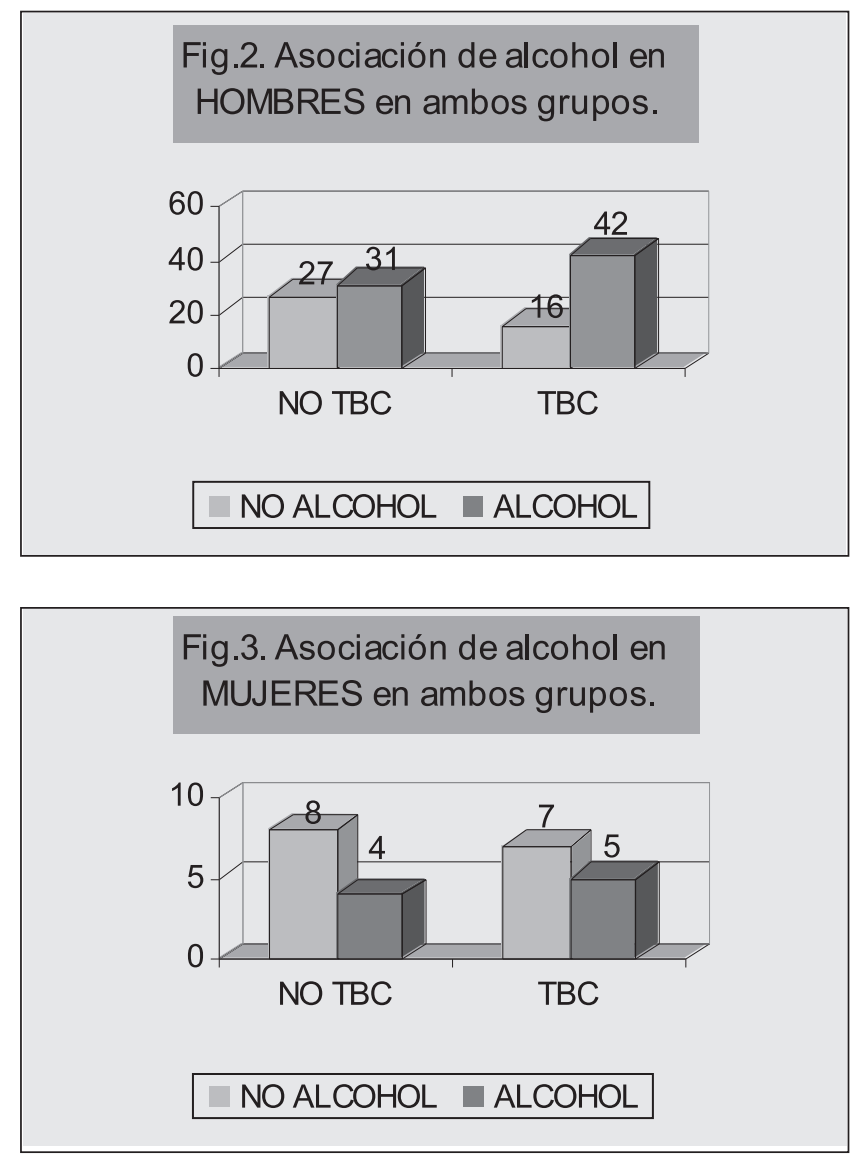

3. Al estudiar la intensidad de consumo alcohólico en ambos grupos encontramos que en el de TBC hay una media de consumo de 94,04 gr/día y en los NO TBC de 20,05 gr/día (Fig.4). Por sexos, observamos que en los bebedores la media de consumo es superior en los enfermos con TBC, tanto para los hombres $(99,21 \mathrm{gr} /$ día, frente a 21,64 gr/día) ( $p=0,0000)$, como para las mujeres 
(50 frente a 7,75) $(p=0,024)$. Sin embargo en los No Tuberculosos las diferencias de consumo de $+13,89$ gr/día entre hombres y mujeres son estadísticamente significativas (Test de Kuscal-Wallis $p=0,0200$ ), mientras que en los Tuberculosos dichas diferencias $(+49,28)$ no es significativa ( $p=0,0712$ ) (Fig.5).
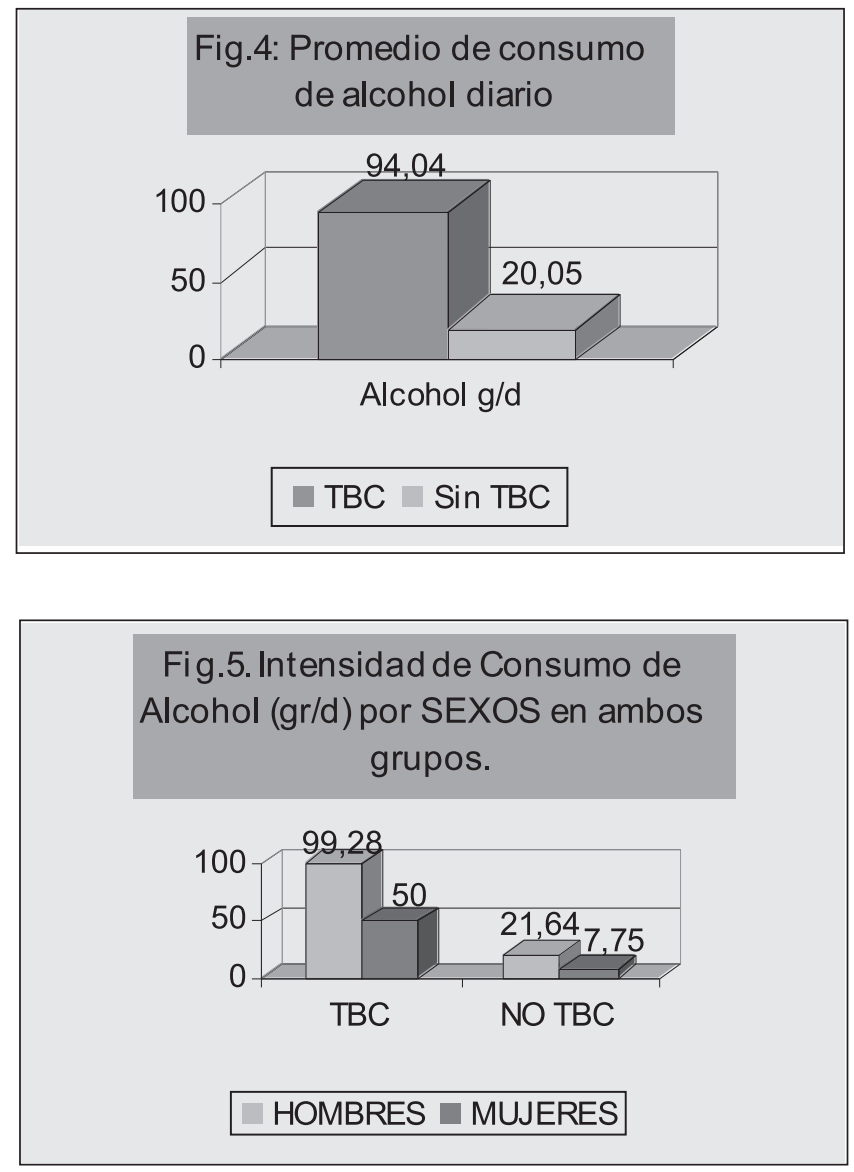

4. Si analizamos el consumo de alcohol en los Tuberculosos, separándolos en dos grupos, casados y no casados (solteros, separados y viudos), encontramos que en el primer grupo (casados), beben alcohol 9/23, y no beben 14/23; mientras que en el grupo de los no casados, beben $38 / 47$ y no beben 9/47. ( $p=0,00048)$.(Fig.6).

Fig.6. Consumo de Alcohol según estado civil en TBC.

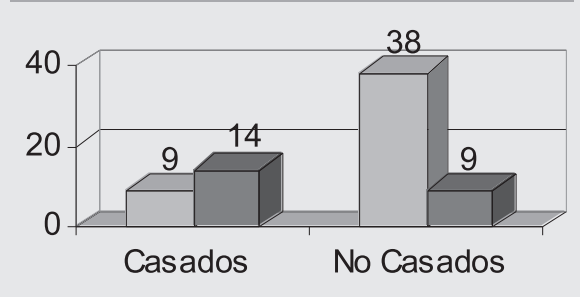

Alcoholico No Alcoholico

\section{CONCLUSIONES / DISCUSIÓN}

1. El consumo de Alcohol se asocia a Tuberculosis pero sólo en los hombres (¿sesgo de respuesta?), aunque convendría estudiar el caso índice y la pareja sexual de nuestras pacientes.

2. Entre los bebedores la intensidad de consumo es mayor en los enfermos de TBC.

3. Entre los enfermos de TBC no hay diferencias de intensidad de consumo por sexo, mientras que en los no tuberculosos, los hombres consumen más cantidad de alcohol.

4. Hay una asociación clara entre TBC y alcohol en el grupo de los no casados (solteros, separados, viudos). Idea que refuerza la necesidad de intervención psicológica y social en éstos estratos, para facilitar la cumplimentación del tratamiento y evitar las recidivas.

\section{BIBLIOBRAFÍA}

1. M. Mata Cases y J. Pallarés Robles. Infecciones frecuentes en Atención Primaria. De: A. Martín Zurro/J.F. Cano Pérez. ATENCION PRIMARIA. Ediciones Mosby/Doyma

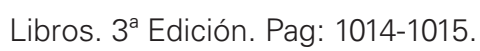

2. Caminero Luna, JA. Tuberculosis: Una perspectiva actual. Jano 23-29 Marzo 2001. VOL. LX N 1.380, 46-47.

3. Cook, RT. Alcohol abuse, alcoholism, and damage to the immune system-a review. Alcohol Clin Exp Res, 1998 Dec, 22:9, 1927-42.

4. Thomsen, JL. Diseases of the airways and lungs in forensic autopsy material of alcoholics. Med Sci Law, 1997 Jan, 37:1, 23-6.

5. M.I. Fernández et al.- Prevalencia de bebedores de riesgo y factores asociados en varones que acu-den a las consultas de Atención Primaria. En: Atención Primaria. Vol. 17. Núm. 3. 29-Febrero-1996.

6. E. Portella et al.- Costes sanitarios del alcoholismo. En: Atención Primaria. Vol 22. Núm.3. 30-Septiembre-1998.

7. F. Escobar et al.- Investigación del consumo de alcohol y diagnóstico de los pacientes alcohólicos en Atención Primaria. En. Atención Primaria. Vol 15. Núm 1. Enero 1995.

8. J.M. Prieto de Paula et al. Tuberculosis Laríngea. Descripción de tres casos. Revista Clínica Espa-ñola. Vol. 195. Núm.4. Abril 1995.

9. J.M. García Páez et al. Tuberculosis en 1991. Estudio Hospitalario. Revista Clínica Española. Vol.193. Núm.5. Octubre 1993. 
